\title{
Real time x-ray studies of rapidly annealed epitaxial layers
}

Walter Lowe

AT\&T Bell Laboratories, Murray Hill, New Jersey 07974

Roy Clarke and Waldemar Dos Passos

Department of Physics, University of Michigan, Ann Arbor, Michigan 48109

Brian Rodricks and Christine Brizard

Advanced Photon Source, Argonne National Laboratory, Argonne, Illinois 60439

(Presented on 16 July 1991)

Time-resolved $x$-ray scattering studies of epitaxial overlayers are presented. The results illustrate the usefulness of high-brightness synchrotron probes for studying the cooperative kinetics of interfaces during rapid thermal processing.

\section{INTRODUCTION}

High-resolution $\mathrm{x}$-ray diffraction is finding increasing application as a reliable nondestructive method for the characterization of epitaxial semiconductors; layer thicknesses, composition, strain, and coherence can be determined with excellent precision. ${ }^{1}$ This information is important in view of the direct bearing of structural quality of heterointerfaces on the electrical characteristics of modern semiconductor devices. With synchrotron sources, it is now feasible to extend diffraction measurements into the time domain. For the present study, we have combined developments in dispersive optics and fast area detectors to obtain measurements while the structure is undergoing changes during the annealing process. ${ }^{2}$

\section{EXPERIMENTAL METHODS}

The kinetics measurements are carried out using a radiation-hardened charge coupled device (CCD) x-ray detector $^{3}$ consisting of $340 \times 580$ pixels, each $22.5-\mu \mathrm{m}$ square (Fig. 1). In order to collect real-time data, a horizontal slit is used to mask the surface of the CCD chip. After a predetermined exposure time $(\approx 100 \mathrm{~ms}$ in this experiment), the charge collected on the exposed area is transferred to the masked area of the chip using the parallel row-transfer register. In this mode, the detector operates as a "streak camera" recording one-dimensional diffraction information with a time-resolution determined by the speed of the parallel transfer $(\approx 20 \mu \mathrm{s} /$ row in the present detector). The CCD is oriented such that the horizontal angular dispersion of the beam is utilized to record a moderately high-resolution ( $\Delta \omega=10$ arc $\mathrm{s})$ diffraction profile.

In a reciprocal space representation (Fig. 2) the scattering geometry is equivalent to a rocking curve (i.e., the length of the scattering wave vector is essentially fixed and its direction sweeps through an angular range of $\approx 250 \mathrm{arc}$ s). Whereas the detector in a standard double-crystal rocking curve geometry is essentially wide open and integrating at each rocking angle, in the dispersive geometry used with an area detector, each incoming ray on the sample is diffracted into a different pixel on the CCD array (Fig. 2).

\section{RESULTS AND DISCUSSION}

Here, we present one example of how these new synchrotron techniques allow us to address the kinetics of strained layer relaxation. We have studied in real time the dynamics of $\mathrm{Ge}_{x} \mathrm{Si}_{1-x}(x \approx 0.20)$ alloy films grown ${ }^{4}$ epitaxially on a silicon (100) substrate. Rather than viewing relaxation as the result of single dislocation kinetics ${ }^{5}$ (nucleation and multiplication), we find that it is necessary to take a global view of the problem. In particular, we take advantage of the power of real-time, $x$-ray scattering to provide information about the long-range cooperative behavior of the strained layer during the relaxation process.

The samples used in this study are $\approx 2000 \AA$ thick and their compositions span the so-called "metastable" region where the kinetics of strain relaxation has been found previously to play an important role in determining the nature of the epitaxial structure. ${ }^{6}$ By monitoring the diffraction angle of the (004) $\mathrm{Ge}_{x} \mathrm{Si}_{1-x}$ peak, which is a direct measure of the perpendicular lattice spacing $b_{1}$, both lattice constants $b_{\perp}$ and $b_{\|}$are known throughout the heating cycle. Since the $\mathrm{x}$-ray penetration depth is several microns, the Si substrate lattice constant $a_{0}$ is also monitored. In this experiment, the tetragonal distortion or strain of the overlayer can be followed in real time during a rapid thermal annealing (RTA) cycle (Fig. 3). Also shown in Fig. 3 for comparison is the calculated lattice constant of a $\mathrm{Ge}_{0.20} \mathrm{Si}_{0.80}$ free-standing film undergoing thermal expansion. This curve represents the behavior that a $\mathrm{Ge}_{0.20} \mathrm{Si}_{0.80}$ film would have if removed from the substrate.

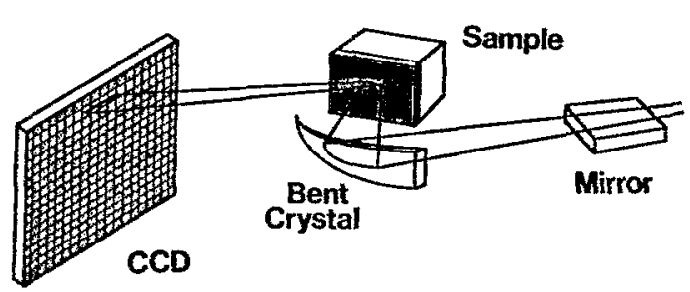

FIG. 1. Experimental arrangement for angular dispersive real-time, $x$-ray scattering studies. The curved crystal is asymmetric-cut $\mathrm{Ge}(111)$. The arrangement corresponds to the configuration of the X-16B AT\&T Bell Labs beamline at the National Synchrotron Light Source, Brookhaven National Laboratory. 
STANDARD ROCKING CURVE

(a)

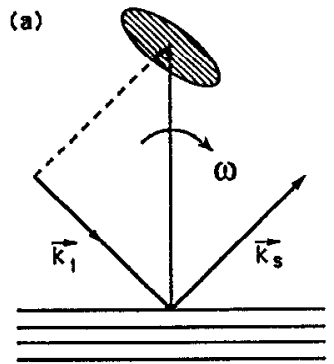

ANGULAR DISPERSED

(b)

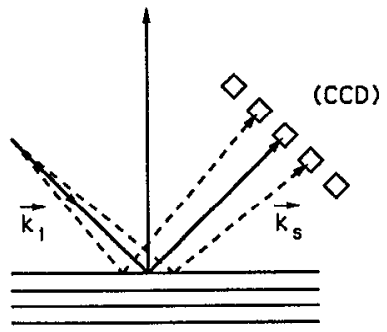
ROCKING CURVE

FIG. 2. Comparison (a) of standard rocking curve geometry with (b) angular dispersive geometry using $\mathrm{CCD}$ array detector. The shaded region in (a) corresponds to the wide acceptance cone of the scintillator detector.

The topmost curve represents the calculated lattice constant $\left(b_{\perp}\right)$ for an ideally pseudomorphic layer.

The most striking feature of Fig. 3 is the existence of several discontinuities. On heating, $b_{1}$ starts increasing along the pseudomorphic thermal expansion curve but then undergoes a transition to a smaller (baseline) value somewhat lower than that at ambient temperature. Then, $b_{1}$ increases, following the same slope as the pscudomorphic curve. On further heating $b_{\perp}$ drops back to its baseline value, increases again, drops back, and so on. A similar behavior, in reverse, is observed on cooling.

During rapid heating the $\mathrm{Ge}_{0.20} \mathrm{Si}_{0.80}$ layer expands along the [001] direction (and also in the basal plane) increasing the vertical strain $b_{1}-b_{\|}$faster than the basal plane expansion. The $\mathrm{Ge}_{0.20} \mathrm{Si}_{0.80}$ basal plane is pinned to the Si lattice by the coherent matching of the lattices at the interface. As $b_{1}-b_{\|}$becomes critical, the $\mathrm{Ge}_{0.20} \mathrm{Si}_{0.80}$ strained layer can reduce its vertical strain as well as continue its expansion in the [001] direction by unpinning and slipping along the interface. Once this occurs, the strain

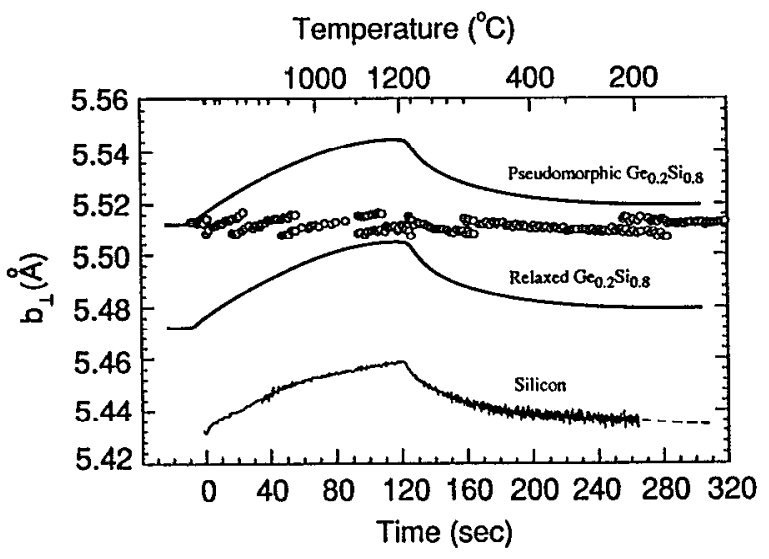

FIG. 3. Perpendicular lattice spacing, $b_{1}$, of a $\mathrm{Ge}_{0.20} \mathrm{Si}_{0.80}$ strained layer (open circles) measured during rapid thermal annealing. Note the discontinuities in $b_{1}$ as the overlayer slips relative to the $\mathrm{Si}$ substrate. The three solid curves are the lattice spacings of the ideal coherently-strained $\mathrm{Ge}_{0.20} \mathrm{Si}_{0.80}$ epitaxial overlayer (top curve), fully relaxed $\mathrm{Ge}_{0.20} \mathrm{Si}_{0.80}$ (middle curve), and the silicon substrate (bottom curve). along [001] is decreased abruptly. A sharp discontinuity in $b_{1}$ accompanied by coexistence clearly indicates that two scattering domains exist concurrently. Note that $b_{1}$ changes by a constant discrete amount $(\approx 0.006 \AA)$ at each discontinuity. This corresponds to a sudden expansion (or contraction) of a coherent region of characteristic size $(8500 \AA)$ by one lattice spacing $(\approx 5.4 \AA)$, parallel to the substrate. Evidence for a coherent microstructure on this length scale has been seen in plan-view $\operatorname{TEM}^{7}$ data on similar films.

In a further set of kinetics experiments, we looked at RTA effects on ion-implanted layers. Specifically, we investigated the effect of ion implantation on the annealing kinetics of $\operatorname{In}_{x} \mathrm{Al}_{1-x}$ As/InP prototype heterostructure insulated gate field effect transistor (HIGFET) layers. Of particular interest is the quality of the layer structure, especially after a rapid thermal annealing treatment used for implant activation. We found that there are dramatic differences in the structural coherence after RTA depending on whether the layers are virgin or implanted.

For these studies, an undoped $5250 \AA$-thick $\mathrm{In}_{x} \mathrm{Al}_{1}-x \mathrm{As}(x=0.54)$, layer was grown by molecular beam epitaxy on a semi-insulating (SI) InP substrate; details of the growth are given in Ref. 8. Si ions were implanted by a double energy $(E)$ process under similar conditions that apply to HIGFET technology, namely $E_{1}$ $=30 \mathrm{keV}$ at dose $D_{1}=2 \times 10^{13} \mathrm{~cm}^{-2}$ followed by $E_{2}$ $=70 \mathrm{keV}, D_{2}=4 \times 10^{13} \mathrm{~cm}^{-2}$. RTA (capless annealing under $\mathrm{Ar}$ gas flow) at $750^{\circ} \mathrm{C}$ for $30 \mathrm{~s}$ was used for implant activation.

Figure 4(a) compares the rocking curves of the virgin (unimplanted) sample before and after RTA. There is essentially no difference. In Fig. 4(b), we again compare the $\mathrm{x}$-ray profiles of the sample before and after RTA, this time in the implanted state; whereas there was virtually no change in the virgin sample, the implanted structure now becomes much more coherent and uniform as the result of an identical RTA treatment. Note the appearance of a well defined fringe pattern [Fig. 4(b)] which fits almost exactly the calculated dynamical scattering ${ }^{9}$ profile for the ideal structure; indeed the value of $\epsilon_{\perp}$ after RTA in implanted samples is almost exactly what is expected ( $\epsilon_{\perp}$ $=0.416 \%$ ) for a perfectly pseudomorphic overlayer at this composition (including the tetragonal distortion) and so there is no dislocation formation during RTA. One possible explanation for the improvement of the long-range coherence in the structure is that the efficiency of coupling the RTA light source into the overlayer may be enhanced by the presence of interband states created by implantation. This would lead to enhanced absorption in the infrared region together with increased heating rates and, consequently, more effective annealing of defects.

In Fig. 5(a), we show the variation in position of the InAlAs overlayer peak measured using real-time synchrotron radiation techniques as the sample is undergoing RTA. Note that, unlike the strained $\mathrm{Ge}_{x} \mathrm{Si}_{1-x}$ case described above, the InAlAs layer is practically lattice matched and unstrained. On heating, we observe a differential thermal expansion. On cooling, a reverse differential 

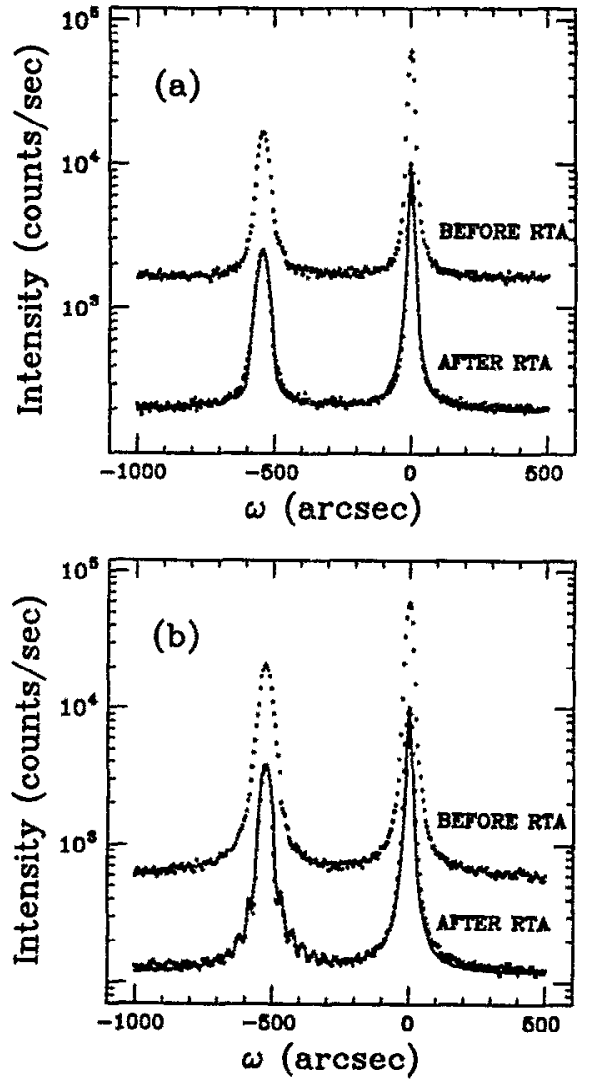

FIG. 4. (a) (400) double-crystal, x-ray rocking curves (dots) of an unimplanted $\mathrm{In}_{0.54} \mathrm{Al}_{0.46}$ As epitaxial layer $(5280 \AA$ ) on InP before and after RTA. The solid line represents a dynamical fit ${ }^{9}$ to the $x$-ray data. (b) (400) rocking curves of the implanted sample before and after RTA. Note the distinet formation of the pendellösung fringes only after RTA.

thermal contraction process is observed before the InAlAs peak position settles into a seemingly stable equilibrium position. Surprisingly, however, a discontinuous transition takes place at $\approx 220 \mathrm{~s}$. This is accompanied by a sharpening of the overlayer peak as the coherence of the overlayer suddenly improves. Figure 5(b) shows another plot of the position of the InAlAs (400) peak in the rocking curve as the sample is subjected to a second RTA process. This time, we observe that no further lattice relaxation has taken place and that the InAlAs peak has attained its stable position characteristic of pseudomorphic growth.

In summary, the measurements obtained in a timeresolved mode by specially developed synchrotron radiation techniques reveal an unexpectedly complex pathway of interfacial kinetics by means of which an entire semiconductor heterostructure can cooperatively attain its ideal, stable state. In particular, we have shown that interfacial strain relaxation is not, as previously believed, a gradual process occurring through local misfit dislocation migration but a discontinous, cooperative mechanism
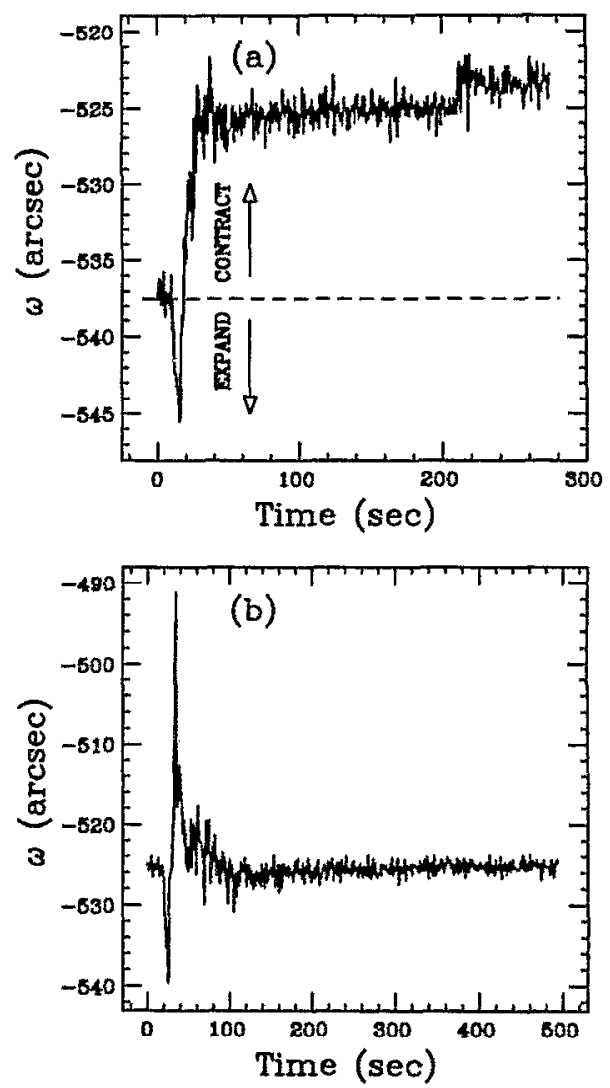

FIG. 5. (a) x-ray peak position of an epitaxial $\mathrm{In}_{0.54} \mathrm{Al}_{0,46} \mathrm{As}$ ( $5280 \AA$ ) layer on InP at the CCD detector as a function of time during RTA, (b) $x$-ray InAlAs peak position at the CCD detector as a function of time as the same sample undergoes a second RTA.

which proceeds via a sequence of metastable states characterized by domains of differing strain.

\section{ACKNOWLEDGMENTS}

The authors wish to acknowledge the support of the Army Research Office (URI Program) under contract DAAL-03-87-K0007 and NSF Grant No. DMR 8805156. We thank Yi-Jen Chan and John Bean, respectively, for growing the InAlAs and $\mathrm{Si} / \mathrm{Ge}$ samples used in this study.

${ }^{1}$ M. H. Lyons and M. A. G. Halliwell, Inst. Phys. Conf. Ser. 76, 445 (1985); L. Tapfer and K. Ploog, Phys. Rev. B 33, 5565 (1986).

${ }^{2}$ R. Clarke, W. Dos Passos, W. Lowe, B. G. Rodricks, and C. Brizard, Phys. Rev. Lett. 66, 317 (1991).

${ }^{3}$ B. Rodricks, R. Clarke, R. Smither and A. Fontaine, Rev. Sci. Instrum. 60, 2586 (1989).

${ }^{4}$ J. C. Bean, T. T. Sheng, L. C. Feldman, A. T. Fiory, and R. T. Lynch, Appl. Phys. Lett. 44, 109 (1984).

${ }^{5}$ S. S. Iyer and F. K. LeGoues, J. Appl. Phys. 65, 4693 (1989).

${ }^{6}$ R. Hull, J. C. Bean, D. J. Werder, and R. E. Leibenguth, Phys. Rev. B 40, 1681 (1989).

${ }^{7}$ R. Hull and J. C. Bean, J. Vac. Sci. Technol. A 4, 2580 (1989).

${ }^{8}$ R. Clarke, W. Dos Passos, Y. J. Chan, and D. Pavlidis, Appl. Phys. Lett. 58, 2267 (1991).

${ }^{9}$ M. J. Hill, B. K. Tanner, M. A. G. Halliwell, and M. H. Lyons, J. Appl. Cryst. 18, 446 (1985). 